\title{
Original Article, Bone SPECT/CT.
}

\section{Role of MDP SPECT/CT Bone Scintigraphy in}

\section{Evaluation of Patients with Malignant Otitis Externa Compared to HRCT: A prospective Study.}

\section{Mostafa, $\mathrm{NM}^{1}$. Salem, $\mathrm{MA}^{2}$. Zidan, $\mathbf{M}^{3}$.}

${ }^{1}$ Department of Clinical Oncology and Nuclear Medicine. ${ }^{2}$ Department of Otorhinolaryngology. ${ }^{3}$ Department of Diagnostic and Interventional Radiology, Faculty of Medicine, Assiut University, Egypt.

\section{ABSTRACT:}

Background: Malignant otitis externa (MOE) is a potentially fatal disease; accurate assessment of the extent of this disease is warranted to plan the course of treatment and to predict the prognosis. The aim of the study was to compare the efficiency of high resolution computed tomography (HRCT) with that of ${ }^{99 \mathrm{~m}}$ Technetium methylene diphosphonate $\left({ }^{99 \mathrm{~m}} \mathrm{Tc}-\mathrm{MDP}\right)$ single photon emission computed tomography/computed tomography (SPECT/CT) in precise evaluation of the disease extent and to evaluate the prognostic value of SPECT/CT staging in terms of therapeutic response and survival in patients with MOE. Patients and Methods: This prospective study included twenty-five patients (17 males and 8 females) with clinically diagnosed MOE, all of them underwent both HRCT and ${ }^{99 \mathrm{~m}} \mathrm{Tc}-\mathrm{MDP}$ SPECT/CT imaging of the skull. Based on ${ }^{99 \mathrm{~m}} \mathrm{Tc}-\mathrm{MDP}$ uptake in SPECT/CT scan, the findings were staged accordingly and compared with the findings of HRCT in all patients. In addition, clinical outcome and disease-free survival (DFS) were analyzed according to the SPECT/CT staging.

Results: Out of 25 patients included in our study, HRCT showed osseous changes in only 4/25 (16\%) patients, ${ }^{99 \mathrm{~m}} \mathrm{Tc}-\mathrm{MDP}$ SPECT/CT demonstrated more osseous involvement in $21 / 25$ (84\%) patients. No patients in stage I in this work, 8 patients had disease confined to the mastoid/temporal bone, not reaching midline (stage II), 6 of them were surviving with recovery and clinical improvement, and 2 patients died. 
Three patients had petrous involvement reaching midline (stage III), 2 of them were surviving with clinical improvement, and the $3^{\text {rd }}$ patient died. Fourteen patients had sphenoid involvement/disease extended to the contralateral temporal bone (stage IV), 5 of them were surviving with recovery and clinical improvement, 5 were surviving, yet had disease progression, and 4 patients died within less than one year of diagnosis. At the end of follow up period; in total; 7 patients had died, of whom 3 in stage IV died due to MOE, while the remaining 4 cases ( 2 in stage II, 1 in stage III, and 1 in stage IV) died due to unrelated causes. Both of disease duration and SPECT/CT staging were statistically significant predictor factors for DFS $(\mathrm{P}=0.05$ and 0.047 respectively).

Conclusion: SPECT/CT has a higher sensitivity than HRCT in determining the exact extent of MOE and was much better correlated with disease prognosis.

Key Words: Malignant otitis externa, skull base osteomyelitis, SPECT/CT, Bone scan, CT imaging.

Corresponding author: Nadia M Mostafa, MD. E-mail: nadia.khalifa@med.aun.edu.eg.

\section{INTRODUCTION:}

Malignant otitis externa (MOE), also referred to as skull base osteomyelitis (SBO) or necrotizing otitis externa (NOE) is a rare serious life-threatening condition ${ }^{(1)}$. It usually commences as infection of the soft tissues of the external auditory canal $(\mathrm{EAC})^{(2)}$, then it can spread through the fissures of Santorini and the tympanomastoid suture line to involve the skull base and temporal bone. The infection can also spread through the venous channels, facial planes, or dural sinuses, eventually reaching the petrous apex ${ }^{(3)}$.
In advanced cases, infection may cross the midline to involve the contralateral temporal

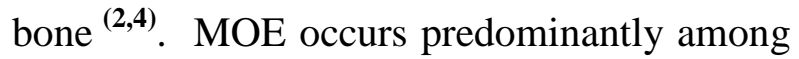
elderly patients with uncontrolled diabetes or immune-compromised status. Pseudomonas aeruginosa is the most commonly isolated microorganism; less commonly; it is caused by Aspergillus fumigatus ${ }^{(2,5)}$. MOE frequently presents as unremitting otalgia, which is often severe, becoming worse at night, and radiating to the ipsilateral face. 
Purulent otorrhea accompanied by oedema of the EAC and granulation tissue or polyp at the bony-cartilaginous junction is pathognomonic for this condition ${ }^{(3)}$.

The Patients may also present with cranial nerve deficits, which can be caused by necrosis, neurotoxins or compression ${ }^{(6)}$. The facial nerve is the first and the most commonly affected cranial nerve due to its proximity to the EAC ${ }^{(7)}$. The exact assessment of the extent of the disease is crucial to decide the duration of treatment and to expect the therapeutic response. Radionuclide and radiologic investigations play an essential role in confirming bone involvement as well as assessing the extent of the disease in the skull base ${ }^{(3)}$. Magnetic resonance imaging (MRI) is the superior imaging modality in identifying the exact anatomical site and extent of soft tissue involvement. However, it can't detect cortical osseous destruction ${ }^{(8)}$. The most frequently used imaging modality for diagnosis and follow-up of skull base osteomyelitis by ENT clinicians is high resolution computed tomography (HRCT) ${ }^{(8,9)}$. Yet, CT needs at least $30 \%$ of the affected bone to be demineralized to pick up disease, so early findings are limited to softtissue inflammation, while skull base bone destruction will be evident only in advanced disease ${ }^{(10)}$. $\quad{ }^{99 m}$ Technetium methylene diphosphonate ( $\left.{ }^{99 \mathrm{~m}} \mathrm{Tc}-\mathrm{MDP}\right) \quad$ bone scintigraphy is more sensitive than radiologic imaging and can detect osseous involvement much earlier than radiologic investigations (11) . Despite ${ }^{99 \mathrm{~m}} \mathrm{Tc}-\mathrm{MDP}$ bone scintigraphy is cost-effective and widely available when compared to Ga-67 scan in the initial assessment of MOE, yet it is not suitable for monitoring treatment response due to persistence of tracer uptake for an indefinite period after an infection resolve ${ }^{(12)}$. In contrast, Ga-67 uptake normalizes after resolution of infection, so it was deemed a superior measure of therapeutic response ${ }^{(\mathbf{1 3})}$. Lee et al (2008) stratified MOE using ${ }^{99 m}$ TcMDP into stages, stage I: Minimal uptake consistent with soft tissue inflammation; stage II: Uptake limited to the mastoid/temporal bone without reaching the midline; stage III: Uptake involving petrous bone and reaching the midline; and stage IV: Uptake involving sphenoid or crossing the midline to the contralateral temporal bones (14) 
In our institution, ENT clinicians depend mainly on HRCT for evaluation of MOE, which led us to do this study in an attempt to clarify the efficacy of MDP-Single photon emission computed tomography/CT (SPECT/CT) scan versus that of HRCT imaging in the assessment of the extent of skull base involvement in patients with MOE and to evaluate the prognostic value of SPECT/CT staging in terms of therapeutic response and survival in these patients.

\section{PATIENTS AND METHODS:}

This prospective study included 25 patients who were clinically diagnosed to have MOE and attended the Otolaryngology department at our institution between April 2018 to August 2019. Ethical clearance for the study was obtained from the institutional Medical Research Ethics Committee. Consent (informed and written) was obtained from each patient.

All patients were subjected to history taking, general and ENT examination, otoscopic and oto-endoscopic examination, taking in consideration the following:

\section{History of:}

A) Diabetes or other immuno-compromised states and its duration.
B) Symptoms: Pain or headache in temporal or pre-auricular and posterior auricular areas, discharge in EAC, hearing loss, and symptoms related to cranial nerve paralysis.

2. Clinical Examination for: Presence of oedema, polyp or granulation tissue in the EAC, perforation of the tympanic membrane, facial and or other cranial nerve paralysis.

3. Audiometric Examination: pure tone air and bone conduction audiogram were recorded at the frequencies of 250, 500, 1000, 2000, 4000, and $8000 \mathrm{~Hz}$ by using a Madson Orbiter 922 diagnostic audiometer by audiologist.

4. Routine laboratory investigations for diabetic patients.

\section{Radiological studies:}

Patients were referred for ${ }^{99 \mathrm{~m}} \mathrm{TC}-\mathrm{MDP}$ SPECT/CT bone scintigraphy and HRCT imaging. HRCT was done in most cases within 2 to 3 days following hospitalization. The 2 imaging modalities were done with a time gap didn't exceed 2-3 weeks. An experienced radiologist (20-year experience) reviewed the HRCT imaging of the all included patients to precisely detect the extent of osseous and soft tissue changes in the skull base. 


\section{Treatment Protocol:}

All patients were hospitalized and treated by adequate diabetes control and parenteral ceftazidime $(1 \mathrm{~g})$ twice daily for 2 weeks and later discharged from the hospital with advice to continue on oral quinolones for 6 weeks. Granulation tissue in the bony canal was excised / cauterized as an office procedure for most patients.

\section{SPECT/CT acquisition:}

SPECT/CT of the skull was performed for all patients in supine position at 2-3 hours after ${ }^{99 \mathrm{~m}}$ Tc-MDP injection (18-22 mci). SPECT images were acquired in a step-and-shoot mode (25 s/ stop), with 32 frames/ head using a non-circular orbit, over $360^{\circ}$ arc $\left(180^{\circ}\right.$ per head), and the image acquisition matrix was $128 \times 128$. Then a low-dose CT scan, for attenuation correction/anatomical localization, was acquired with a peak tube voltage of $130 \mathrm{kV}, 80 \mathrm{~mA}$ tube current, and 512x512 matrix. SPECT images were reconstructed using iterative reconstruction algorithm (Flash 3D, Siemens Healthcare, Erlangen, Germany) with 4 iterations, 4 subsets and $8 \mathrm{~mm}$ Gaussian filter. Transverse, sagittal, and coronal slices were generated. CT images were reconstructed with slice thickness of $1 \mathrm{~mm}$, then SPECT images were hardware coregistered and fused with CT images, and the resulting fused series were checked and corrected for any displacement to ensure optimal coregistration. Finally, reconstructed SPECT/CT images were obtained in the transverse, sagittal, and coronal planes.

\section{HRCT acquisition:}

The HRCT was done using 64 detector scanners (Aquilion CT; Toshiba Medical Systems). A helical acquisition is done for temporal bone imaging and routinely use a collimation of $0.625 \mathrm{~mm}$ section thickness, $0.5 \mathrm{~s}$ rotation time, FOV $15-20 \mathrm{~cm}$, highresolution matrix $(512 \times 512)$ in bone algorithm, $140 \mathrm{kV}$ and $300 \mathrm{~mA}$. Scanning was done in axial plane and the head is kept in neutral position and true axial images parallel to Reid's line (joining inferior margin of the orbit to the center of the orifice of EAC) are acquired.

For the axial technique, the patient is supine positioned. The scan is then planned for acquisition from the lateral scout topogram from the arcuate eminence through the mastoid tip. Coronal multi-planar reconstruction is made perpendicular to the axial scans. The images were reconstructed and visualized in special bone algorithm. 
Interpretation of SPECT/CT: Based on radiotracer uptake, we stratified SPECT/CT findings into stages according to the prognosis-based classification suggested by Lee et al. (2008).

Stage I: Minimal radiotracer uptake.

Stage II: Radiotracer uptake limited to the mastoid/temporal bone without reaching the midline.

Stage III: Radiotracer uptake involved the petrous part of temporal bone reaching, yet not crossing the midline.

Stage IV: Radiotracer uptake that was involving sphenoid bone or crossing the midline and extending into the contralateral temporal bone

\section{Statistics:}

Data were verified, coded by the researcher, and analyzed using IBM-SPSS version $24^{*}$. Descriptive statistics: Means, standard deviations, medians, interquartile range (IQR), and percentages were calculated. Test of significances: chi-square test was used to compare the difference in distribution of frequencies among different groups. For continuous variables with more than two categories; ANOVA test was calculated to test the mean differences of the data that follow normal distribution and independent sample Kruskal-Wallis was used to compare the median difference between groups that didn't follow normal distribution. Disease free survival (DFS) was calculated as the time from the onset of disease to the time of last follow up or confirmed event (disease progression or death). Disease duration was calculated as the time from the onset of disease to the time of presentation. Survival analysis was performed using Kaplan-Meier test to estimate the median survival time. The difference between the categories of the explanatory variables was compared using Log-rank test. A p-value equal or less than 0.05 was considered statistically significant.

\section{RESULTS:}

\section{Clinico-demographic characteristics.}

This prospective study included 25 patients with clinically diagnosed MOE. Out of them, 17 were males $(68 \%)$ and 8 females $(32 \%)$. Their mean age at presentation was 60.52 \pm 7.1 years (range: $48-73$ years), and the mean disease duration at the time of presentation was $3.32 \pm 0.5$ months. 
All of them were diabetic, had unilateral ear disease, and presented with characteristic severe otalgia of MOE. Twenty-four cases presented with hearing loss (7 conductive, 7 sensorineural, and 10 mixed hearing loss) and otorrhea. On clinical examination at the time of presentation, granulation tissue in the EAC was present in 21 patients, tympanic membrane perforation in 5 patients, and parotid swelling in one patient. Six patients presented with cranial nerve paralysis (5 of them had facial nerve paralysis and the remaining one had vagus nerve paralysis) (Table 1).

Table (1): Clinical and demographic characteristics of 25 patients.

\begin{tabular}{|c|c|c|c|}
\hline \multicolumn{2}{|c|}{ Parameters } & No & $\%$ \\
\hline \multirow[b]{2}{*}{ Sex } & Male & 17 & 68 \\
\hline & Female & 8 & 32 \\
\hline \multirow[b]{2}{*}{ Affected Side } & Right & 12 & 48 \\
\hline & Left & 13 & 52 \\
\hline \multirow{7}{*}{$\begin{array}{l}\text { Medical history \& clinical } \\
\text { presentation }\end{array}$} & Diabetes & 25 & 100 \\
\hline & Otalgia & 25 & 100 \\
\hline & Otorrhea & 24 & 96 \\
\hline & Hearing loss & 24 & 96 \\
\hline & Parotid swelling & 1 & 4 \\
\hline & External canal granulation & 21 & 84 \\
\hline & Tympanic membrane perforation & 5 & 20 \\
\hline \multirow[t]{2}{*}{ Cranial neuropathy } & Facial nerve & 5 & 20 \\
\hline & Vagus nerve & 1 & 4 \\
\hline
\end{tabular}




\section{SPECT/CT staging and its association with demographic, clinical characteristics, and outcome.}

We didn't have any patients with minimal radiotracer uptake; so; our study didn't include stage I disease. Eight patients had stage II disease (Figure 1). Three patients had stage III disease (Figure 2).

The remaining 14 patients were recorded to have stage IV disease (Figure 3).

SPECT/CT of three patients demonstrated radiotracer uptake at further osseous sites in addition to the above mentioned documented skull base lesions; at the root of left zygoma in one case which was included into stage II, left occipital condyle and left jugular foramen in the $2^{\text {nd }}$ and right jugular foramen in the $3^{\text {rd }}$ case, both of the $2^{\text {nd }}$ and $3^{\text {rd }}$ cases were included into stage IV (Figure 4).

Patient with stage II had higher age, yet without statistical significance $(\mathrm{P}=0.513)$. Duration of DM had no statistical difference among different stages $(\mathrm{P}=0.879)$. Cranial neuropathy was significantly evident in advanced stages (III \& IV), duration of
MOE was significantly longer in stage III, and male predominance was significantly more frequently seen in stages II \& IV $(\mathrm{P}=0.030,0.028 \& 0.015$, respectively). Full recovery was significantly associated with stage II, while mortality rate was significantly higher in both stages III \& IV $(\mathrm{P}=0.049)$ (Table 2).

All the studied patients were followed up for a period ranged from 1-28 months (mean, 12.52 \pm 1.40 months), the shortest follow up was related to the early death of a patient in stage IV. For stage II: Six patients were surviving, 5 of them achieved full recovery and the $6^{\text {th }}$ had clinical improvement; while the remaining 2 patients died after 2 and 13 months of diagnosis. For sage III: Two patients were surviving with clinical improvement, while the $3^{\text {rd }}$ patient died after 20 months of diagnosis.

For stage IV: Ten patients were surviving, 3 of them achieved full recovery, 2 were improved, and 5 had disease progression; while the remaining 4 patients died within less than one year of diagnosis (at 1, 2, 3, and 9 months). 
Table (2): Association of SPECT/CT staging with demographic, clinical characteristics, and long-term outcome of the study population.

\begin{tabular}{|c|c|c|c|c|c|}
\hline \multicolumn{2}{|c|}{ Characteristic } & Stage II & Stage III & Stage IV & $\mathbf{P}$ \\
\hline \multicolumn{2}{|l|}{ Age/years \pm SD } & $62.75 \pm 9.1$ & $57.67 \pm 8.7$ & $59.86 \pm 5.5$ & $0.513 *$ \\
\hline \multirow[b]{2}{*}{ Sex } & Male & $8(100 \%)$ & $1(33.3 \%)$ & $8(57.1 \%)$ & \multirow{2}{*}{$\begin{array}{c}\text { Cramer's } \\
\mathrm{V}=0.497 \\
P=0.015^{*} *\end{array}$} \\
\hline & Female & $0(0 \%)$ & $2(66.7 \%)$ & $6(42.9 \%)$ & \\
\hline \multirow{2}{*}{$\begin{array}{l}\text { Cranial } \\
\text { neuropathy }\end{array}$} & yes & $0(0 \%)$ & $2(66.7 \%)$ & $4(28.6 \%)$ & \multirow{2}{*}{$\begin{array}{l}\text { Cramer's } \\
\mathrm{V}=0.477 \\
P=0.030 \text { *** }\end{array}$} \\
\hline & No & $8(100 \%)$ & $1(33.3 \%)$ & $\begin{array}{l}11 \\
(78.6 \%)\end{array}$ & \\
\hline \multicolumn{2}{|c|}{ DM duration/years (mean \pm SE) } & $12.13 \pm 2.2$ & $10 \pm 1.2$ & $12.21 \pm 2.1$ & $0.879 * * *$ \\
\hline \multicolumn{2}{|c|}{ Disease duration (mean \pm SE) } & $3.25 \pm 0.65$ & $7.33 \pm 2.3$ & $2.5 \pm 0.4$ & $0.028 * * *$ \\
\hline \multirow{4}{*}{ Outcome } & Recovery & $5(62.5 \%)$ & $0(0 \%)$ & $3(21.4 \%)$ & \multirow{4}{*}{$\begin{array}{l}\text { Cramer's } \\
\mathrm{V}=0.479 \\
P=0.049 * *\end{array}$} \\
\hline & Improvement & $1(12.5 \%)$ & $2(66.7 \%)$ & $2(14.3 \%)$ & \\
\hline & Progression & $0(0 \%)$ & $0(0 \%)$ & $5(35.7 \%)$ & \\
\hline & Death & $2(25 \%)$ & $1(33.3 \%)$ & $4(28.6 \%)$ & \\
\hline
\end{tabular}

* ANOVA test was used to compare the mean difference between groups.

**Chi-square test with likelihood ratio was used to compare the difference in frequency between groups, Cramer's V for strength of association between nominal and ordinal variables.

***Kruskal Wallis test was used to compare the mean ranks between groups, data expressed as mean $\pm \mathrm{SE}$. 
Egyptian J. Nucl. Med., Vol. 22, No. 1, June 2021

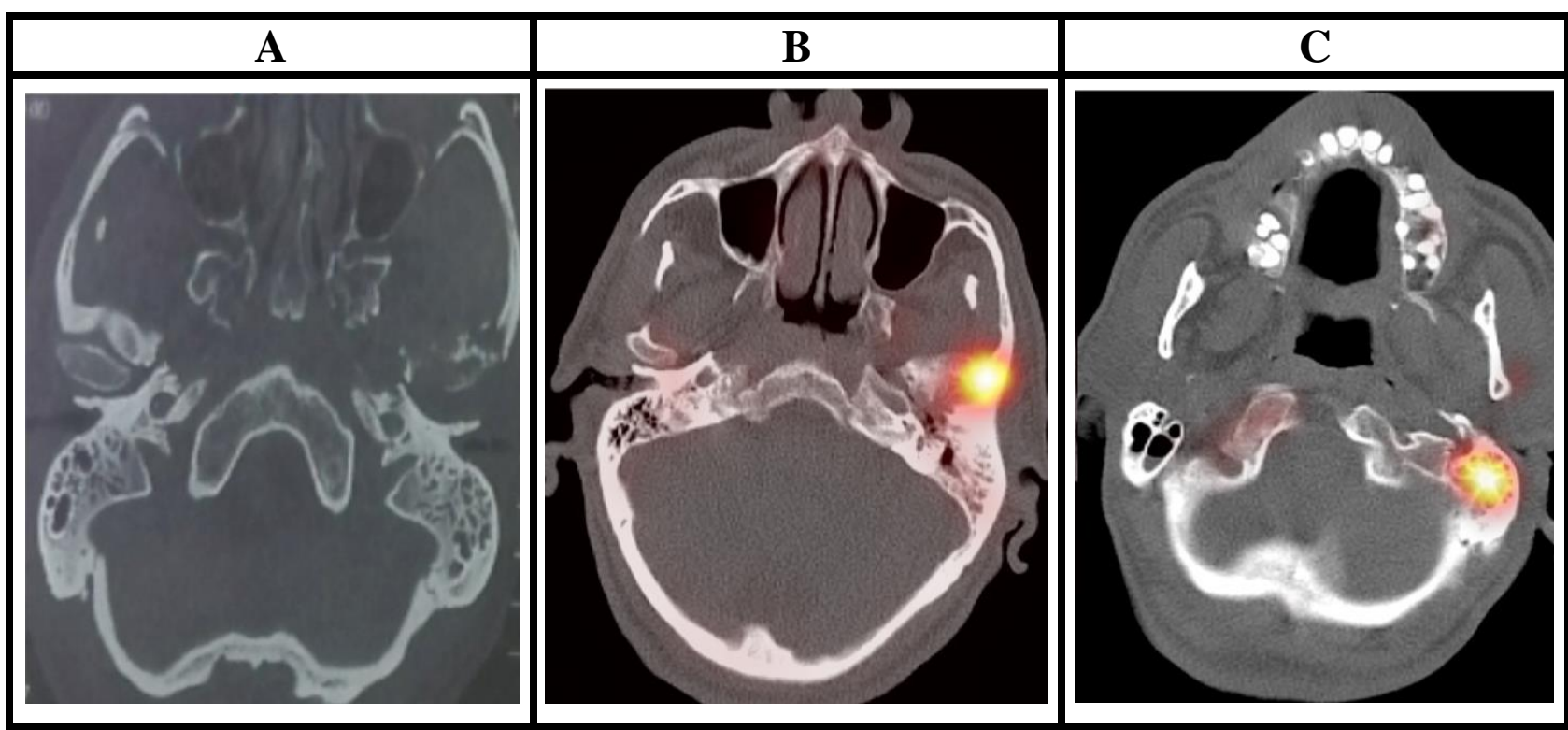

Figure (1): A 48-year old diabetic male, presented with left sided hearing loss, otalgia and persistent purulent otorrhea refractory to antibiotics. The clinical examination show end granulation tissue of the external auditory canal.

(A) HRCT images showed obvious osteolysis of the root of left zygoma.

(B\&C) SPECT/CT images demonstrated active tracer uptake at the root of left zygoma and the left mastoid temporal bone, respectively (stage II).

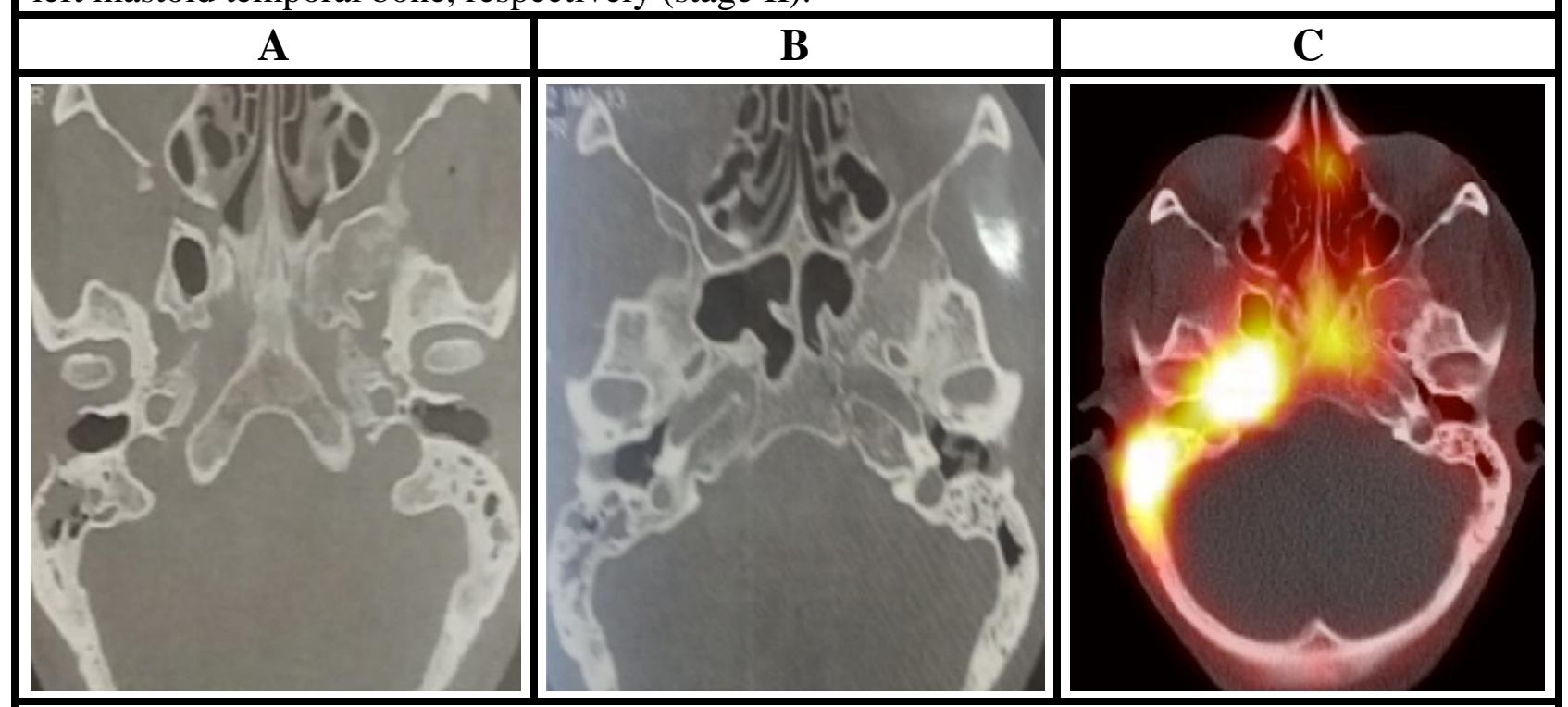

Figure (2): A 65-year old diabetic female, presented with right otalgia, purulent otorrhea and facial nerve palsy. The clinical examination showed granulation tissue of the external auditory canal.

(A\&B) HRCT images showed right mastoid opacity, yet it didn't reveal any evidence of bone erosion in the right temporal bones, while (C) SPECT/CT images demonstrated active tracer uptake at the mastoid and petrous portions of the right temporal bone reaching the midline (stage III). 
Egyptian J. Nucl. Med., Vol. 22, No. 1, June 2021

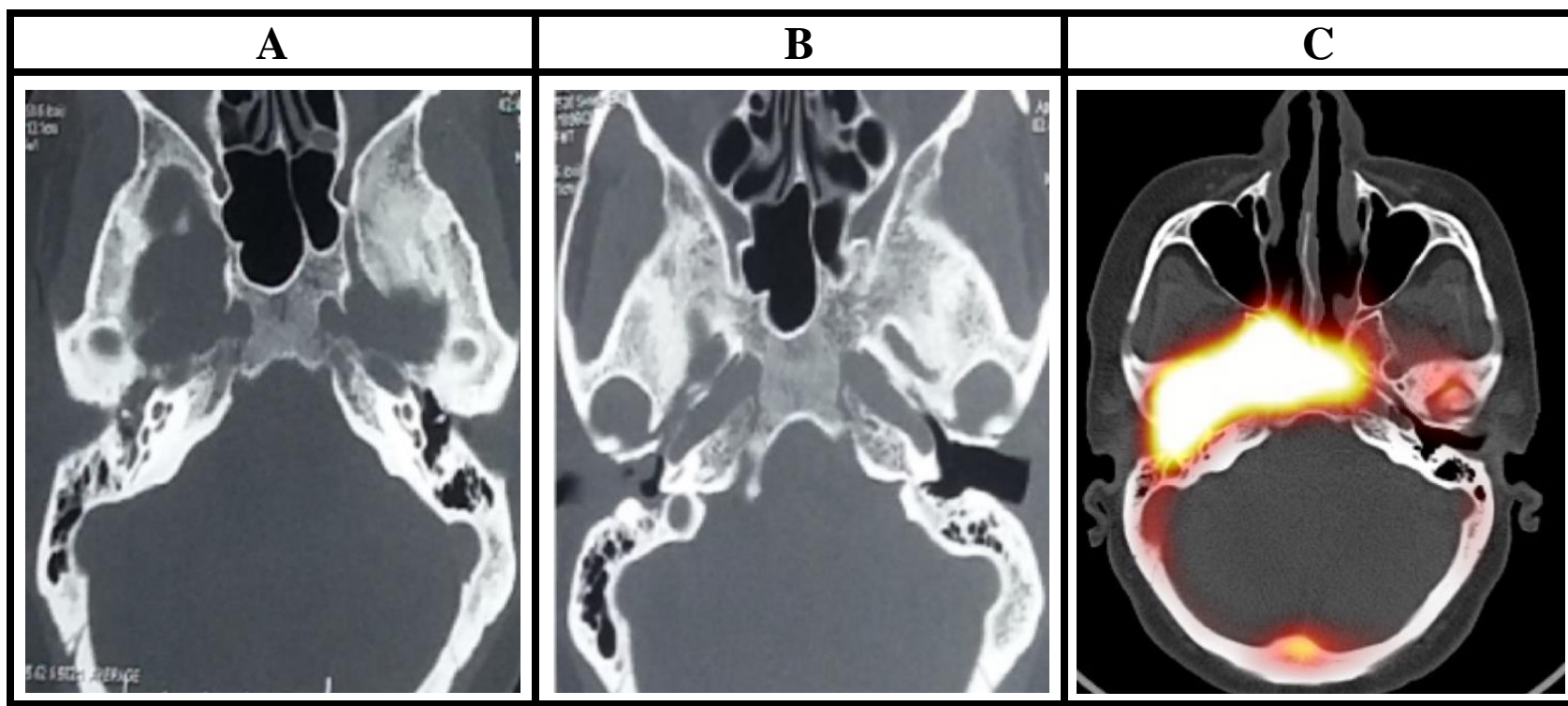

Figure (3): A 57-year old diabetic female, presented with right sided hearing loss, otalgia and purulent otorrhea. The clinical examination showed oedema and granulation tissue of the external auditory canal. (A\&B) HRCT images showed no evidence of bone erosion in the right temporal, clivus or sphenoid bones, while (C) SPECT/CT images demonstrated active tracer uptake at the right temporal, clivus, sphenoid bones and crossing the midline to the contralateral temporal bone (stage IV)

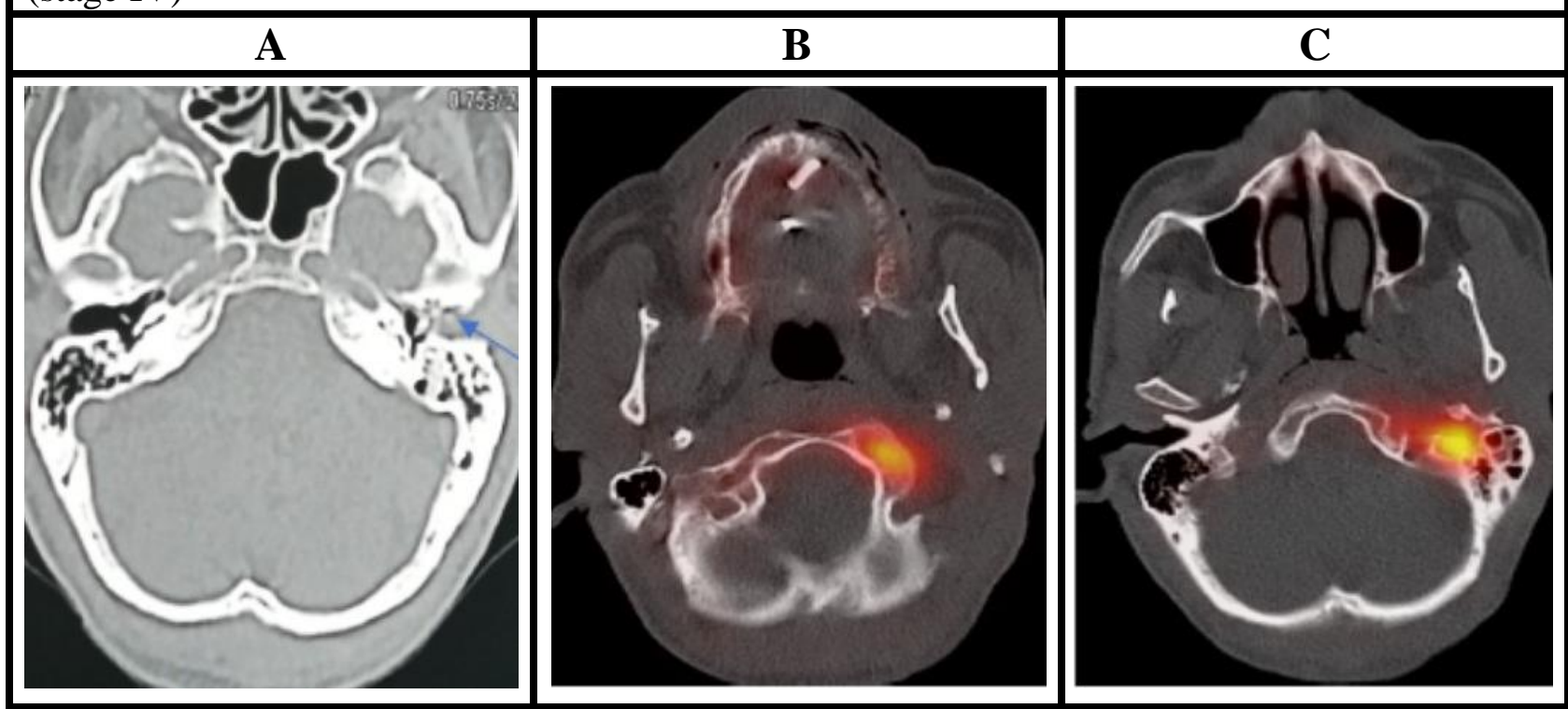

Figure (4): A 62-year old diabetic male, presented with left sided hearing loss, otalgia and purulent otorrhea. The clinical examination showed granulation tissue of the left external auditory canal and perforation of the left tympanic membrane.

(A) HRCT images showed erosions of the left bony external auditory canal (arrowed). $(B \& C)$ SPECT/CT images demonstrated active tracer uptake at the left occipital condyle and left jugular foramen, respectively (stage IV). 


\section{HRCT findings in relation to SPECT/CT staging:}

HRCT revealed soft tissue changes in $22 / 25$ (88\%) patients; which involved all patients in stage II and stage III (100\%), and 11/14 (78.6\%) patients in stage IV. Opacification of mastoid air cells was found in 21/25 (84\%) patients. However; only 4/25 (16\%) patients had evidence of bone erosion in HRCT; one was stage II (12.5\%), the second was stage III (33.3\%), and the remaining 2 patients were stage IV (14.3\%). Thus, SPECT/CT demonstrated more osseous involvement in 21/25 (84\%) patients (Table 3).

Table (3): Comparison of SPECT/CT staging with HRCT findings.

\begin{tabular}{|l|c|c|c|}
\hline SPECT/CT Stage & \multirow{2}{*}{ No. (25) } & \multicolumn{2}{|c|}{ HRCT findings, No. (\%) } \\
\cline { 3 - 4 } & & Soft tissue changes & Bony erosions \\
\hline Stage II & 8 & $8(100)$ & $1(12.5)$ \\
\hline Stage III & 3 & $3(100)$ & $1(33.3)$ \\
\hline Stage IV & 14 & $11(78.6)$ & $2(14.3)$ \\
\hline Overall & 25 & $22(88)$ & $4(16)$ \\
\hline
\end{tabular}

\section{Univariate Cox proportional hazard regression analysis for DFS:}

On univariate Cox proportional hazard regression analysis for DFS, among the studied variables, we demonstrated that disease duration and SPECT/CT staging were the only factors significantly impacted the DFS $($ Hazard ratio $(\mathrm{HR})=0.675,95 \% \mathrm{CI}$ :
0.451-0.997, $\mathrm{P}=0.05)$ and $(\mathrm{HR}=7.74,95 \% \mathrm{CI}$ : 1.002-22.45, $\mathrm{P}=0.048$ ) respectively.

Other variables (patient's age and sex, cranial neuropathy, and duration of DM) were tested but found not to be significantly associated with DFS (Table 4). 
Egyptian J. Nucl. Med., Vol. 22, No. 1, June 2021

Table (4): Univariate Cox proportional hazard regression analysis for DFS.

\begin{tabular}{|l|c|c|}
\hline \multirow{2}{*}{ Variable } & \multicolumn{2}{|c|}{ Univariate } \\
\cline { 2 - 4 } & HR (95\% CI) & P \\
\hline Age/years & $0.979(0.901-1.06)$ & 0.6 \\
\hline Sex (Female) & $2.003(0.634-6.33)$ & 0.2 \\
\hline Cranial Neuropathy & $1.146(0.302-4.348)$ & 0.8 \\
\hline $\begin{array}{l}\text { Disease } \\
\text { Duration/month }\end{array}$ & $0.675(0.451-0.997)$ & 0.05 \\
\hline DM Duration/years & $0.993(0.891-1.11)$ & 0.9 \\
\hline SPECT/CT Staging & $* 7.74(1.002-22.45)$ & 0.048 \\
\hline
\end{tabular}

HR, Hazard Ratio; CI, Confidence Interval * Hazard Ratio of stage IV.

\section{Association between the duration of DFS and SPECT/CT staging:}

We found a significant difference in the duration of DFS among different SPECT/CT stages, patients in stage III had the highest median of DFS (29 month), followed by stage
II patients (22 months), while stage IV patients had the lowest median of DFS (13 months), (log rank=7.479, $p=0.024)$ (Table 5 \& Figure 5). 
Egyptian J. Nucl. Med., Vol. 22, No. 1, June 2021

Table (5): Means and Medians of DFS according to SPECT/CT stages.

\begin{tabular}{|l|c|c|c|c|c|}
\hline SPECT/CT staging & $\begin{array}{c}\text { Mean DFS } \\
\text { duration. } \\
\text { months }\end{array}$ & SE & 95\% CI & $\begin{array}{c}\text { Median DFS } \\
\text { duration. } \\
\text { months }\end{array}$ & SE \\
\hline Stage II & 23.800 & 2.496 & $18.907-28.693$ & 22.000 & 3.708 \\
\hline Stage III & 28.667 & 3.538 & $21.732-35.601$ & 29.000 & 2.81 \\
\hline Stage IV & 15.857 & 3.200 & $9.585-22.130$ & 13.000 & 3.367 \\
\hline Overall & 20.796 & 2.413 & $16.067-25.525$ & 20.000 & 3.286 \\
\hline Overall & Log rank=7.479, $p=0.024$ & & \\
\hline
\end{tabular}

Data expressed as mean \pm SE, median \pm SE, $95 \%$ confidence interval, Kaplan-Meier for analysis.

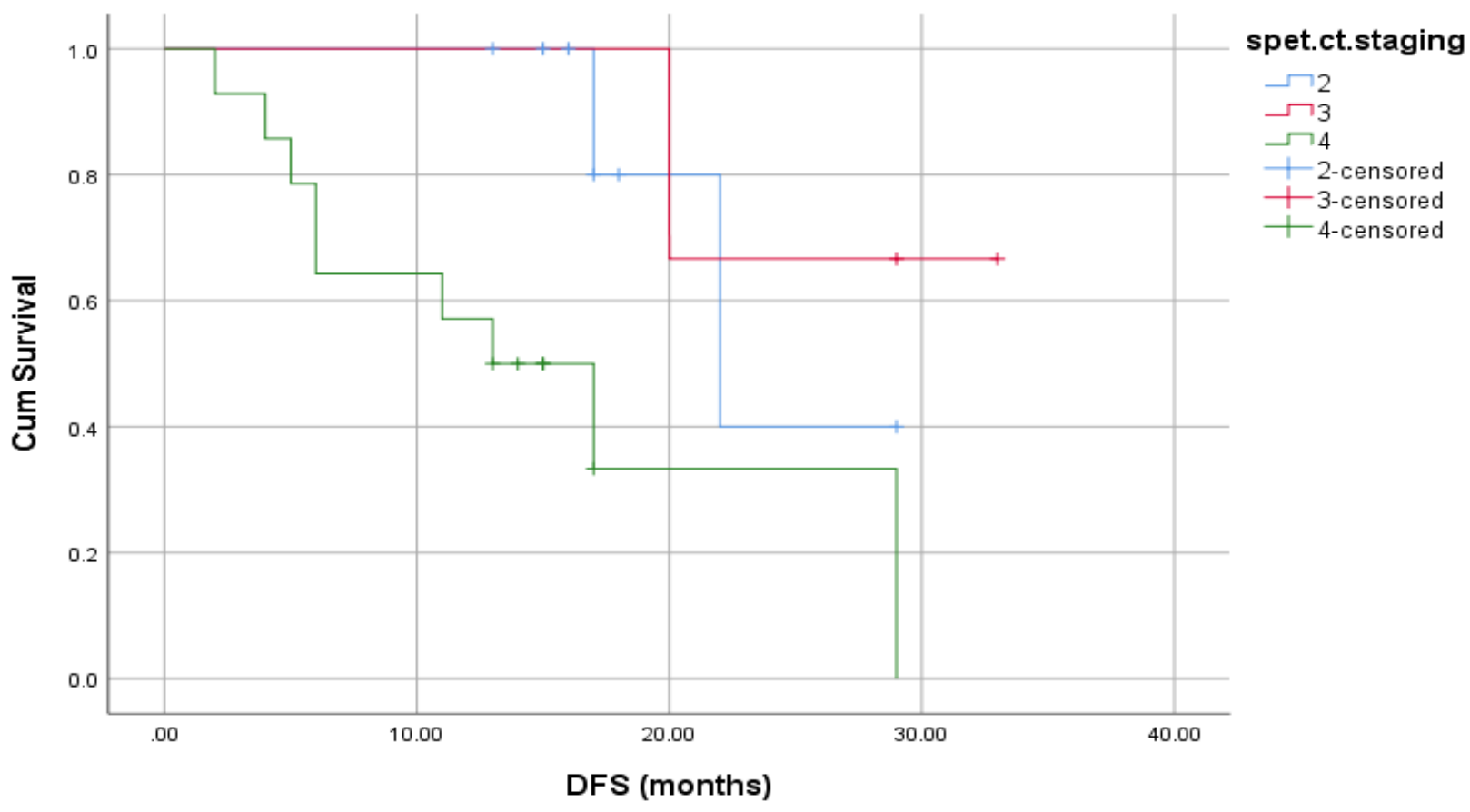

Figure (5): DFS according to different SPECT/CT stages. 


\section{DISCUSSION:}

Malignant otitis externa (MOE), is an aggressive infection of the EAC which invades beyond its confines into the adjacent tissues and typically into the skull base ${ }^{(15)}$. It is associated with high disease specific mortality ${ }^{(13)}$. In our study, all the included patients were diabetic; it is unclear why MOE is much more common in elderly diabetics. A combination of impaired poly-morpho nuclear cell function and micro-angiopathy along with increased $\mathrm{pH}$ of cerumen in the aural canal of diabetics likely makes them more vulnerable to invasive infection ${ }^{(15)}$.

The mean duration of DM prior to the diagnosis of MEO for the studied patients was $11.92 \pm 6.70$ years, albeit without significant association with DFS or significant difference between different SPECT/CT stages $(\mathrm{P}=0.9$ and 0.879, respectively). Chen and Hsieh (2013), also suggested that the duration of DM didn't affect survival ${ }^{(11)}$.

In agreement with relevant reports, we found a significant association between sex and incidence of MOE with a male predominance $(68 \% \text { males Vs. } 32 \% \text { females })^{(\mathbf{1 , 3 , 1 6})}$, yet again without significant impact on DFS $(\mathrm{P}=0.2)$.
The results of the current study demonstrated that MOE can be prognostically staged at presentation. Clinical staging depending on the nerve involvement, while the radiological staging on the disease extension; stage I encompasses disease confined to the EAC with or without facial nerve palsy, stage II encompasses disease invasion of the skull base and/or involvement of multiple cranial nerves, while intracranial involvement is categorized as stage III ${ }^{(17)}$. We reported 6 patients $(24 \%)$ with cranial nerve involvement ( 5 of them had facial nerve palsy), which is comparable to the reported percentage (24-43\%) of cranial nerve involvement in other studies ${ }^{(\mathbf{1 8})}$. Furthermore, we found a significant association between cranial neuropathy and SPECT/CT staging, it was more frequent in higher stages $(\mathrm{P}=0.030)$, yet without a statistically significant relationship to the outcome $(\mathrm{P}=0.8)$. Also, a previous study done by Lee et al. (2017) analyzing the prognostic factors in MOE didn't reveal significant difference in the outcome of MOE with respect to any specific cranial nerve or multiple cranial nerves involvement ${ }^{(7)}$. 
HRCT scanning is a fast and cost-effective imaging tool in the initial assessment of patients with MOE. Effacement of fat planes in the sub-temporal area and subtle cortical bone erosions are the initial findings ${ }^{(19)}$. However, for bone erosion to be evident on CT, more than $30 \%$ of the affected bone must be demineralized ${ }^{(20)}$. Furthermore, Sudhoff et al. (2008) reported that $\mathrm{CT}$ had a limited value in predicting the outcome of $\mathrm{MOE}^{(21)}$.

${ }^{99 \mathrm{~m}}$ Tc- MDP accumulates in remodeling bone by incorporation into the crystalline structure of calcium hydroxyapatite ${ }^{(22)}$. Increased MDP uptake reflects the increased bone turnover, it is very sensitive to detect even $10 \%$ rise in osteoblastic activity ${ }^{(23)}$. Moreover, ${ }^{99 \mathrm{~m}} \mathrm{Tc}$ MDP scan can identify any abnormalities of bone metabolism as early as $24-48$ hours after the onset of pathology ${ }^{(24)}$.

Previous studies documented that ${ }^{99 \mathrm{~m}} \mathrm{Tc}$ - MDP bone scintigraphy has high sensitivity in the diagnosis of MOE and that infection can be detected even when the CT does not detect osseous erosion ${ }^{(25,26)}$. However, despite being a sensitive tool for diagnosis of osteomyelitis, conventional ${ }^{99 \mathrm{~m}}$ Tc-MDP planar scintigraphy cannot accurately detect the site of bone involvement in MOE especially the centrally located lesions due to its limited spatial resolution ${ }^{(27)}$.
After the advent of SPECT/CT, wherein SPECT is superimposed on CT images, it allowed better identification of the disease extent $^{(3) \text {. }}$

It is worth to be mentioned that the current study was not designed to evaluate the added value of SPECT/CT over planar imaging for diagnosis of MOE, as many previous studies had reported that SPECT/CT is more accurate than planar imaging in detecting SBO $(11,16,27,28,29)$, and therefore little added value might be gained from further comparative studies between both entities.

Chen \& Hsieh (2013) and Sharma et al. (2013) recommended the use of MDP SPECT/CT as a routine investigation in evaluation of $\mathrm{MOE}^{(\mathbf{1 1}, 28)}$.

To the best of our knowledge, there were only two studies that had been carried out to evaluate the role of ${ }^{99 \mathrm{~m}} \mathrm{Tc}-\mathrm{MDP}$ SPECT/CT and SPECT in staging /grading of MOE ${ }^{(3,14)}$, respectively.

Balakrishnan et al. (2019) in a similar recent prospective study compared the efficacy of HRCT to that of SPECT/CT in the assessment of disease stage and found that HRCT underestimated the real stage of the disease as it demonstrated bony changes in only 8 (28.6\%) of 28 studied population ${ }^{(3)}$. 
Matching with these results, our study revealed low percentage of HRCT detected bony changes; positive osseous findings were only found in 4/25 (16\%) of patients.

On the other hand, Lee et al. (2008) performed HRCT for 24/38 patients included in their study but didn't find evidence of bony erosion in any of them. Furthermore, we noticed that their SPECT based stratification system broadly staged MOE according to the aforementioned definite parts of the skull base without allowing flexibility to include additional sites $^{(\mathbf{1 4})}$.

The present study revealed further sites of osseous extension; root of zygoma, occipital condyle, and jugular foramina in 3 cases; which were categorized into stage II for the first lesion and stage IV for the last 2 lesions, as we hypothesized that the peripheral/lateral skull located zygomaticus and the central/medial skull located occipital/jugular foramina bony lesions had the same prognostic significance as that of stage II and stage IV skull base lesions, respectively. Relying on SPECT only in their stratification system could explain this limited flexibility 14), which further advocates the incremental value of hybrid SPECT/CT compared to SPECT in the precise localization of skull osseous lesions in MOE.

Regarding disease outcomes, the authors found that SPECT grading, fungal/mixed infection, cranial neuropathy, immune status, and Charlson score of comorbidity were significant predictors for DFS on univariate analysis, yet SPECT grading was the only factor which remained independently associated with survival on multivariate analysis.

So, they suggested that SPECT-based grading system of MOE accurately predicted the longterm prognosis of the studied patients ${ }^{(\mathbf{1 4})}$.

For ours, among the studied variables, we demonstrated that SPECT/CT staging as well as the duration of MOE were the only prognostic factors associated with DFS on the univariate cox regression analysis $(\mathrm{P}=0.048 \&$ 0.05 respectively).

In our study, 7 patients died during their follow up; 4 of them were stage IV; rapid disease progression was the underlying etiology for death in 3 of them, while the remaining patient in stage IV and the other 3 ( 2 in stage II and 1 in stage III) patients died due to unrelated causes.

So, death due to MOE as well as disease progression was absolutely recorded for stage IV. 
Moreover, patients in stage IV had the lowest mean of DFS, promoting the significance of ${ }^{99 \mathrm{~m}}$ Tc-MDP SPECT/CT staging in predicting the outcome of MOE. On the other hand, we found that patients in stage III had the highest mean of disease duration, yet they had the highest mean of DFS. The few number of patients included in this category along with the long follow up duration for this stage could explain this unexpected finding.

The powerful points of the current work included its prospective design, expanding of the previously proposed SPECT based disease stratification to involve further skull base and extra skull base lesions, and including disease duration along with other various variables in the analysis of prognostic factors predicting the outcome of MEO.

\section{Limitations of study:}

Small sample size due to the rarity of disease resulted in very small number of the included patients in stage III, with subsequent unexpected statistical results. Also, absence of culture of the discharged ear liquid for most of the studied patients, because almost all of them started antibiotic treatment before hospitalization. Furthermore, Ga-67 scan hadn't been conducted to monitor the disease progress because of its low availability and its high cost. Lastly, we didn't assess the responsiveness to anti-diabetic treatment which was believed to be correlated with the disease outcome ${ }^{(30)}$. So, larger prospective multicenter studies are needed for further verification of the association between SPECT/CT staging and long-term outcome of patients with MOE.

\section{CONCLUSIONS:}

SPECT/CT is a more sensitive diagnostic imaging test than HRCT in evaluating the extent of skull base involvement among patients with MOE. Being important to accurately stage such disease in deciding the treatment course and in predicting the outcome, we recommend that SPECT/CT should be performed for all cases diagnosed or suspected to have MOE in addition to HRCT even though HRCT doesn't reveal any osseous changes. 


\section{REFERNCES:}

1. Adams $A$ and Offiah $C$. Central skull base osteomyelitis as a complication of necrotizing otitis externa: imaging findings, complications, and challenges of diagnosis. Clin Radiol, 67 (10): 7-16; 2012.

\section{Sreepada GS and Kwartler JA.}

Skull base osteomyelitis secondary to malignant otitis externa. Curr Opin Otolaryngol Head Neck Surg, 11 (5): 316$23 ; 2003$.

3. Balakrishnan R, Dalakoti P, Nayak DR, et al,. Efficacy of HRCT Imaging vs SPECT/CT Scans in the Staging of Malignant External Otitis. Otolaryngol Neck Surg, 161 (2): 336-42; 2019.

\section{Bernheim $J$ and Sade $J$.} Histopathology of the soft parts in 50 patients with malignant external otitis. J Laryngol Otol, 103 (4): 366-8; 1989.

\section{Shpitzer T, Levy R, Stern Y, et al,.} Malignant external otitis in nondiabetic patients. Ann Otol Rhinol Laryngol, 102 (11): 870-2; 1993.
6. Mahdyoun P, Pulcini C, Gahide I, et al,. Necrotizing otitis externa: a systematic review. Otol Neurotol, 34 (4):620-9; 2013.

7. Lee SK, Lee SA, Seon SW, et al,. Analysis of prognostic factors in malignant external otitis. Clin Exp Otorhinolaryngol, 10 (3): 228; 2017.

8. Van Kroonenburgh A, Van der Meer WL, Bothof RJP, et al,. Advanced imaging techniques in skull base osteomyelitis due to malignant otitis externa. Curr Radiol Rep, 6 (1): 3; 2018.

9. Chawdhary $G$, Pankhania $M$, Douglas S, et al,. Current management of necrotising otitis externa in the UK: survey of 221 UK otolaryngologists. Acta Otolaryngol, 137 (8): 818-22; 2017.

\section{Murray $M E$ and Britton $J$.} Osteomyelitis of the skull base: the role of high resolution CT in diagnosis. Clin Radiol, 49 (6): 408-11; 1994. 
11. Chen $Y-H$ and Hsieh H-J. Single photon emission computed tomography/computed tomography for malignant otitis externa: lesion not shown on planar image. Am J Otolaryngol, 34 (2):169-71; 2013.

12. Stokkel MPM, Boot ICN, van EckSmit BLF. SPECT Gallium Scintigraphy in Malignant External Otitis: Initial Staging and Follow- up. Case Reports. Laryngoscope, 106 (3): 338-40; 1996.

13. Sturm JJ, Stern Shavit S, Lalwani $\boldsymbol{A K}$. What is the Best Test for Diagnosis and Monitoring Treatment Response in Malignant Otitis Externa? Laryngoscope, 130 (11): 2516-7; 2020.

14. Lee S, Hooper R, Fuller A, et al,. Otogenic cranial base osteomyelitis: a proposed prognosis-based system for disease classification. Otol Neurotol, 29 (5): 66672; 2008.

15. Morales RE, Eisenman DJ, Raghavan P. Imaging Necrotizing Otitis Externa. In: Seminars in roentgenology, 54 (3): 215-26; 2019.
16. Yeddes I, Bahloul A, Meddeb I, et al, Single photon emission computed tomography/computed tomography (SPECT/CT) of the skull in necrotizing otitis externa: comparison with planar bone scintigraphy, SPECT and CT. J Otolaryngol ENT Res, 6 (1):145; 2017.

\section{Okpala NCE, Siraj QH, Nilssen E,} et al,. Radiological and radionuclide investigation of malignant otitis externa. J Laryngol Otol, 119 (1): 71; 2005.

18. Mani N, Sudhoff H, Rajagopal S, et al,. Cranial nerve involvement in malignant external otitis: implications for clinical outcome. Laryngoscope, 117 (5): 907-10; 2007.

19. Curtin HD, Wolfe P, May M. Malignant external otitis: CT evaluation. Radiology, 145 (2): 383-8; 1982.

20. Grandis JR, Branstetter BF, Victor $\boldsymbol{L Y}$. The changing face of malignant (necrotising) external otitis: clinical, radiological, and anatomic correlations. Lancet Infect Dis, 4 (1): 34-9; 2004. 
21. Sudhoff H, Rajagopal S, Mani N, et $\boldsymbol{a l}$,. Usefulness of CT scans in malignant external otitis: effective tool for the diagnosis, but of limited value in predicting outcome. Eur Arch oto-rhino-laryngology, 265 (1): 53-6; 2008.

22. Kanishi D. $99 \mathrm{mTc}-\mathrm{MDP}$ accumulation mechanisms in bone. Oral surgery, oral Med oral Pathol, 75 (2): 23946; 1993.

23. Asimakopoulos $P$, Supriya $M$, Kealey S, et al,. A case-based discussion on a patient with non-otogenic fungal skull base osteomyelitis: pitfalls in diagnosis. J Laryngol Otol, 127 (8): 817; 2013.

\section{Ganhewa $A D$ and Kuthubutheen J.}

A diagnostic dilemma of central skull base osteomyelitis mimicking neoplasia in a diabetic patient. Case Reports, bcr-2012007183; 2013.

25. Chakraborty D, Bhattacharya A, Kamaleshwaran KK, et al,. Single photon emission computed tomography/computed tomography of the skull in malignant otitis externa. Am J Otolaryngol, 33 (1):128-9; 2012.
26. Strashun AM, Nejatheim M, Goldsmith SJ. Malignant external otitis: early scintigraphic detection. Radiology, 150 (2): 541-5; 1984.

27. Younis JA. Additive value of 99mTechnetium methylene diphosphonate hybrid single-photon emission computed tomography/computed tomography in the diagnosis of skull base osteomyelitis in otitis externa patients compared to planar bone scintigraphy. World J Nucl Med, 17 (4): 286; 2018.

28. Sharma P, Agarwal KK, Kumar S, et al,. Utility of $99 \mathrm{~m}$ Tc-MDP hybrid SPECT-CT for diagnosis of skull base osteomyelitis: comparison with planar bone scintigraphy, SPECT, and CT. Jpn J Radiol, 31 (2): 81-8; 2013.

29. Horger M, Eschmann SM, Pfannenberg $C$, et al,. Added value of SPECT/CT in patients suspected of having bone infection: preliminary results. Arch Orthop Trauma Surg, 127 (3): 211-21; 2007.

30. Joshua BZ, Sulkes J, Raveh E, et al,. Predicting outcome of malignant external otitis. Otol Neurotol, 29 (3): 33943; 2008. 
Egyptian J. Nucl. Med., Vol. 22, No. 1, June 2021 